\title{
Parthenolide plays a protective role in the liver of mice with metabolic dysfunction-associated fatty liver disease through the activation of the HIPPO pathway
}

\author{
WEIHONG WANG* ${ }^{*}$ YUKAI HE ${ }^{*}$ and QIULI LIU \\ Department of Hepatology, Liaocheng People's Hospital, Liaocheng, Shandong 252000, P.R. China
}

Received July 16, 2020; Accepted November 20, 2020

DOI: $10.3892 / \mathrm{mmr} .2021 .12126$

\begin{abstract}
Metabolic dysfunction-associated fatty liver disease (MAFLD) is a serious threat to human health. Parthenolide (PAR) displays several important pharmacological activities, including the promotion of liver function recovery during hepatitis. The aim of the present study was to assess the effect of PAR on MAFLD in a mouse model. Body weight, liver to body weight ratios, histological score, alanine transaminase, aspartate transaminase, total cholesterol and triglyceride levels were determined to evaluate liver injury. Liver hydroxyproline concentrations were also assessed. The expression levels of lipid metabolism-related genes (sterol regulatory element binding protein-1c, fatty acid synthase, acetyl CoA carboxylase 1, stearoyl CoA desaturase 1 and carbohydrate response element-binding protein, peroxisome proliferator-activated receptor $\alpha$, carnitine palmitoyl transferase $1 \alpha$ and acyl-CoA dehydrogenase short chain), liver fibrosis-associated genes $(\alpha$-smooth muscle actin, tissue inhibitor of metalloproteinase 1 and TGF- $\beta 1$ ), pro-inflammatory cytokines (TNF- $\alpha$, IL- $1 \beta$ and IL-6) and oxidative stress-associated enzymes (malondialdehyde, superoxide dismutase and glutathione peroxidase) were measured in mice with MAFLD. The expression levels of genes associated with the HIPPO pathway were also measured. In vivo experiments using a specific inhibitor of HIPPO signalling were performed to verify the role of this pathway in the effects of PAR. PAR exerted beneficial effects on liver injury, lipid metabolism, fibrosis, inflammation and oxidative stress in mice with MAFLD, which was mediated by activation of the HIPPO pathway.
\end{abstract}

Correspondence to: Dr Qiuli Liu, Department of Hepatology, Liaocheng People's Hospital, 67 Dongchang West Road, Dongchangfu, Liaocheng, Shandong 252000, P.R. China

E-mail: liuqiuli0211@163.com

${ }^{*}$ Contributed equally

Key words: metabolic dysfunction-associated fatty liver disease, parthenolide, HIPPO pathway, lipid metabolism, inflammation, liver fibrosis

\section{Introduction}

Metabolic dysfunction-associated fatty liver disease (MAFLD), previously known as non-alcoholic fatty liver disease (NAFLD), is the most globally prevalent liver disease linked to the accumulation of fat in the liver (1-4). MAFLD is characterized by histological lesions ranging from steatosis, non-alcoholic steatohepatitis and fibrosis to cirrhosis and hepatocellular carcinoma (5). MAFLD develops as a consequence of improper lifestyle, such as unhealthy diet, sedentary habits and excessive sugar intake, and threatens the health of $25-30 \%$ of the global population $(6,7)$. The main treatment option for MAFLD is weight loss (8). There is no approved pharmacological treatment for MAFLD, due to the complex and diverse pathogenesis of this disease (9). Thus, identification of new approaches for the treatment of MAFLD is required.

Recently, traditional Chinese herbal extracts have been shown to alleviate MAFLD (10). Parthenolide (PAR), an active sesquiterpene lactone extracted from the feverfew plant, can promote the apoptosis of various malignant cell types (11). In mice, PAR treatment can reduce acute hepatitis and promote functional recovery of the liver (12). PAR has also been reported to protect against liver fibrosis (13). Furthermore, a previous study indicated that PAR could represent a candidate agent for MAFLD treatment (14). However, the molecular mechanisms underlying the effects of PAR in MAFLD remain unclear.

A previous study has demonstrated that the HIPPO pathway regulates gene expression and that its mediators, Yes-associated protein (YAP) and transcriptional coactivator with PDZ-binding motif (TAZ), are associated with the development, regeneration and tumorigenesis of the liver (15). The HIPPO pathway is associated with hepatic fibrosis, due to the involvement of oxidative stress injury and macrophage inflammation (16). Additionally, the HIPPO pathway is associated with extracellular matrix stiffness and hepatic fibrosis in hepatic stellate cells (17). HIPPO/YAP signalling also participates in the regulation of lipid metabolism (18). Moreover, the essential role of the HIPPO pathway in the initiation and progression of MAFLD-associated hepatocellular carcinoma has been demonstrated (19).

Therefore, considering the protective role of PAR and involvement of the HIPPO pathway in several liver-associated disorders, it was hypothesized that PAR could display similar 
efficacy in MAFLD, possibly through the HIPPO pathway. Consequently, histological and molecular experiments were carried out to identify the regulatory mechanism governing the role of PAR in MAFLD and to provide novel insight into therapeutic strategies against MAFLD initiation and progression.

\section{Materials and methods}

Reagent preparation. PAR (Sigma-Aldrich; Merck KGaA) and verteporfin (VP) (Selleck Chemicals) were dissolved in DMSO to prepare a $100-\mathrm{mM}$ solution, which was stored at $-30^{\circ} \mathrm{C}$ and diluted to the required concentration using $9 \%$ normal saline.

Animal treatment and grouping. C57BL/6 male mice (age, 8 weeks old; weight, 25-30 g) were purchased from Guangdong Medical Laboratory Animal Centre. Mice were housed at $21 \pm 2^{\circ} \mathrm{C}$ and $50 \pm 5 \%$ relative humidity with a 12 -h day/night cycle and free access to food and drinking water. After one week of adaptation, 144 mice were randomly allocated into 8 groups with 18 mice per group: i) Mice in the control group were fed normal diet and intraperitoneally injected with $200 \mu \mathrm{l}$ normal saline containing $1.5 \%(\mathrm{v} / \mathrm{v})$ DMSO three times a week; ii) mice in the control + PAR group were fed normal diet and intraperitoneally injected with $6 \mathrm{mg} / \mathrm{kg}$ PAR three times a week; iii) mice in the model group were fed high-fat diet and intraperitoneally injected with $200 \mu \mathrm{l}$ normal saline containing $1.5 \%(\mathrm{v} / \mathrm{v})$ DMSO three times a week; iv) mice in the PAR-low $(\mathrm{L})$, medium $(\mathrm{M})$ and high $(\mathrm{H})$ groups were fed high-fat diet and intraperitoneally injected with 2,4 , or $6 \mathrm{mg} / \mathrm{kg}$ PAR three times a week $(20,21)$; v) mice in the PAR-VP group were fed high-fat diet and intraperitoneally injected with $6 \mathrm{mg} / \mathrm{kg}$ PAR three times a week followed by daily injections of $50 \mathrm{mg} / \mathrm{kg}$ VP five weeks later (lasting for three weeks); and vi) mice in the PAR negative control (PAR-NC) group were fed high-fat diet and intraperitoneally injected with $6 \mathrm{mg} / \mathrm{kg}$ PAR three times a week followed by daily injections of $200 \mu 1$ normal saline containing $1.5 \%(\mathrm{v} / \mathrm{v})$ DMSO five weeks later (lasting for three weeks).

Normal mouse diet (cat. no. D12450H; Research Diets, Inc.) and high-fat diet (cat. no. D12451; Research Diets, Inc.) contained 12 and $45 \% \mathrm{kcal}$ fat, respectively. Mice were fed for 8 weeks and weighed every week. At the end of the experiment, $1 \%$ pentobarbital sodium $(45 \mathrm{mg} / \mathrm{kg})$ was intraperitoneally injected in mice for anaesthesia. The mouse eyeballs were extracted for blood collection, and the blood was centrifuged at $900 \mathrm{x} \mathrm{g}$ for $15 \mathrm{~min}$ at $4^{\circ} \mathrm{C}$. The serum was stored at $-20^{\circ} \mathrm{C}$. The mice were then immediately fixed and their thoracic cavity was opened for cardiac perfusion. When the liver completely turned white, sterile surgical instruments were used for liver resection. The liver of each mouse was weighed, and the liver index was calculated (liver weight/body weight) $\mathrm{x} 100 \%$. The livers of 6 mice in each group were combined as a tissue homogenate sample that was stored at $-80^{\circ} \mathrm{C}$. The same regions of the liver of 6 other mice in each group were used to prepare frozen sections $\left(-20^{\circ} \mathrm{C}\right)$. The same regions of the liver of the remaining 6 mice were fixed in $10 \%$ neutral formalin at room temperature for $4 \mathrm{~h}$ to prepare paraffinized sections for tissue staining.
The present study was approved by The Animals Ethics Committee of Liaocheng People's Hospital (approval no. 2019036). Significant efforts were made to minimize the number of animals used and the pain they experienced. All procedures were conducted strictly in accordance with the National Institutes of Health Guide for the Care and Use of Laboratory Animals.

Biochemical analysis and ELISA. The alanine aminotransferase (ALT; cat. no. C009-2-1) and aspartate aminotransferase (AST; cat. no. C010-2-1) levels in the serum were detected according to the instructions of the commercial kits. The supernatant was prepared by centrifugation of the liver tissue homogenate at $16,000 \mathrm{xg}$ for $10 \mathrm{~min}$ at $4^{\circ} \mathrm{C}$. Total cholesterol (TC; cat. no. A111-1-1), triglyceride (TG; cat. no. A110-1-1) and malondialdehyde (MDA; cat. no. A003-1-2) levels and superoxide dismutase (SOD; cat. no. A001-3-2) and glutathione peroxidase (GSH-Px; cat. no. A005-1-2) activities were measured in the liver tissue samples according to the manufacturer instruction for the corresponding kits. All kits were purchased from Nanjing Jiancheng Bioengineering Institute.

Haematoxylin and eosin $(H \& E)$ staining and NAFLD activity score $(N A S)$. Paraffinized sections $(6 \mu \mathrm{m})$ were dewaxed and stained with haematoxylin (Beijing Solarbio Science \& Technology Co., Ltd.) for $3 \mathrm{~min}$ at room temperature. The sections were then washed and placed in hydrochloric acid and ammonia for $2 \mathrm{sec}$ followed by washes with distilled water. Then, the sections were stained with eosin for $5 \mathrm{~min}$ at room temperature and washed with distilled water. Finally, the sections were dehydrated in gradient alcohol, cleared in xylene, and sealed using neutral gum. Sections were observed under a CKX41SF inverted microscope (Olympus Corporation). The NAS was used for histological scoring, liver injury: i) Steatosis (0-3, 0 represents $<5 \%$, and 3 represents $>66 \%)$; ii) lobular inflammation (0-2, 0 represents no foci, and 2 represents 2-4 foci); and iii) hepatocellular ballooning $(0-2,0$ represents none and 2 represents a high number of cells/prominent ballooning) (22).

Oil Red $O$ staining. Frozen sections $(7 \mu \mathrm{m})$ of the liver were fixed in $10 \%$ neutral formaldehyde at room temperature for $30 \mathrm{~min}$ and washed three times with deionized water. Oil Red O solution (Sigma-Aldrich; Merck KGaA) was added at room temperature for $10 \mathrm{~min}$. The sections were then washed with deionized water, stained with haematoxylin at room temperature for $2 \mathrm{~min}$, sealed with neutral gum and observed under a light microscope (Olympus CKX51; Olympus Corporation).

Masson's trichrome staining. Paraffinized sections were dewaxed, stained with $100 \mu 1$ Masson mixture (Beijing Solarbio Science \& Technology Co., Ltd.) for $5 \mathrm{~min}$ and washed using distilled water. The sections were then stained with $100 \mu \mathrm{l}$ phosphomolybdic acid for $5 \mathrm{~min}$ and dried. Then, the sections were stained with $100 \mu 1$ of aniline blue for $5 \mathrm{~min}$ and washed with distilled water. Sections were washed twice continuously (each time for $40 \mathrm{sec}$ ) using $1 \%$ glacial acetic acid $(100 \mu 1)$. Finally, the sections were dehydrated using 95\% alcohol and anhydrous alcohol, cleared, sealed using neutral gum and examined under a light microscope (Olympus 
Table I. Primer sequences for reverse transcription-quantitative PCR.

\begin{tabular}{|c|c|}
\hline Gene & Primer sequence \\
\hline PPAR- $\alpha$ & $\begin{array}{l}\text { F: 5'-ATGGTGGACACAGAGAGCCCCAT-3' } \\
\text { R: 5'-TCAGTACATGTCTCTGTAGAT-3' }\end{array}$ \\
\hline SREBP-1c & $\begin{array}{l}\text { F: 5'-GTGAGCCTGACAAGCAATCA-3' } \\
\text { R: 5'-GGTGCCTACAGAGCAAGAG-3' }\end{array}$ \\
\hline FASN & $\begin{array}{l}\text { F: 5'-GGAGGTGGTGATAGCCGGTAT-3' } \\
\text { R: 5'-TGGGTAATCCATAGAGCCCAG-3' }\end{array}$ \\
\hline $\mathrm{ACC} 1$ & $\begin{array}{l}\text { F: 5'-ATTGGGCACCCCAGAGCTA-3' } \\
\text { R: 5'-CCCGCTCCTTCAACTTGCT-3' }\end{array}$ \\
\hline SCD1 & $\begin{array}{l}\text { F: 5'-TTCTTGCGATACACTCTGGTGC-3' } \\
\text { R: 5'-CGGGATTGAATGTTCTTGTCGT-3' }\end{array}$ \\
\hline CPT1 $1 \alpha$ & $\begin{array}{l}\text { F: 5'-CACCAACGGGCTCATCTTCTA-3' } \\
\text { R: 5'-CAAAATGACCTAGCCTTCTATCGAA-3 }\end{array}$ \\
\hline ChREBP & $\begin{array}{l}\text { R: 5'-ATGCGCGAATACCACAAGTGGAGA-3' } \\
\text { R: 5'-TTATAATGGTCTCCCCAGGGTGC-3' }\end{array}$ \\
\hline ACADS & $\begin{array}{l}\text { F: 5'-GACTGGCGACGGTTACACA-3' } \\
\text { R: 5'-GGCAAAGTCACGGCATGTC-3' }\end{array}$ \\
\hline$\alpha-S M A$ & $\begin{array}{l}\text { F: 5'-GTCCCAGACATCAGGGAGTAA-3' } \\
\text { R: 5'-TCGGATACTTCAGCGTCAGGA-3' }\end{array}$ \\
\hline TIMP-1 & $\begin{array}{l}\text { F: 5'-TTCGTGGGGACACCAGAAGTC-3' } \\
\text { R: 5'-TATCTGGGACCGCAGGGACTG-3' }\end{array}$ \\
\hline TGF- $\beta 1$ & $\begin{array}{l}\text { F: 5'-ATTCCTGGCGTTACCTTGG-3' } \\
\text { R: 5'-AGCCCTGTATTCCGTCTCCT-3' }\end{array}$ \\
\hline TNF- $\alpha$ & $\begin{array}{l}\text { F: 5'-CCTGTAGCCCACGTCGTAG-3' } \\
\text { R: 5'-GGGAGTAGACAAGGTACAACCC-3' }\end{array}$ \\
\hline IL-1 $\beta$ & $\begin{array}{l}\text { F: 5'-ACGGACCCCAAAAGATGAAG-3' } \\
\text { R: 5'-TTCTCCACAGCCACAATGAG-3' }\end{array}$ \\
\hline IL-6 & $\begin{array}{l}\text { F: 5'-CAAAGCCAGAGTCCTTCAGAG-3' } \\
\text { R: 5'-GTCCTTAGCCACTCCTTCTG-3' }\end{array}$ \\
\hline MST1 & $\begin{array}{l}\text { F: 5'-CTTCCACTACAACATGAGCAGC-3' } \\
\text { R: 5'-TGCAGGTCCGCACATAATCTT-3' }\end{array}$ \\
\hline LATS1 & $\begin{array}{l}\text { F: 5'-CGGAGTACTTCAGAAGTTAATCC-3' } \\
\text { R: 5'-TACTGACAGATGATCCTCCTC-3' }\end{array}$ \\
\hline YAP & $\begin{array}{l}\text { F: 5'-CCCGACTCCTTCTTCAAGC-3' } \\
\text { R: 5'-CTCGAACATGCTGTGGAGTC-3' }\end{array}$ \\
\hline TAZ & $\begin{array}{l}\text { F: 5'-TCTGGACCAAGTACATGAACC-3' } \\
\text { R: 5'-AGGACTGGTGATTGGACAC-3' }\end{array}$ \\
\hline$\beta$-actin & $\begin{array}{l}\text { F: 5'-GGCATGGGTCAGAAGGATTCC-3' } \\
\text { R: 5'-ATGTCACGCACGATTTCCCGC-3' }\end{array}$ \\
\hline
\end{tabular}

F, forward; R, reverse; SREBP-1c, sterol regulatory element binding protein-1c; FASN, fatty acid synthase; ACC1, acetyl CoA carboxylase 1; SCD1, stearoyl CoA desaturase 1; ChREBP, carbohydrate response element-binding protein; PPAR- $\alpha$; peroxisome proliferator-activated receptor $\alpha$; ACADS, acyl-CoA dehydrogenase short chain; CPT1 $\alpha$, carnitine palmitoyl transferase $1 \alpha$; $\alpha$-SMA, $\alpha$-smooth muscle actin; TIoMP-1, tissue inhibitor of metalloproteinase 1; MST1, macrophage-stimulating 1; YAP, Yes-associated protein; LATS1, large tumour suppressor kinase 1; TAZ, TAZ, transcriptional coactivator with PDZ-binding motif.
CKX51; Olympus Corporation). All staining assays were performed at room temperature.

Assay of liver hydroxyproline content. Liver hydroxyproline content was detected using a kit (cat. no. A030-2-1; Nanjing Jiancheng Bioengineering Institute) according to the manufacturer's instructions. Liver samples $(20 \mathrm{mg})$ were weighed and hydrolysed using an alkaline solution at $95^{\circ} \mathrm{C}$ for $20 \mathrm{~min}$. Then, $1 \mathrm{ml}$ supernatant was added into $\mathrm{R} 1$ and allowed to stand for $10 \mathrm{~min}, \mathrm{R} 2$ was added for $5 \mathrm{~min}$ and $\mathrm{R} 3$ was added to water bath at $60^{\circ} \mathrm{C}$ for $15 \mathrm{~min}$. A spectrophotometer was used to measure the optical density (OD) at $550 \mathrm{~nm}$, and hydroxyproline content per gram of liver tissue was calculated using a standard curve.

Reverse transcription-quantitative (RT-q) PCR. Total RNA was extracted from liver samples using a MiniBEST Universal RNA extraction kit (Takara Biotechnology Co., Ltd.), and the concentration of RNA was measured. Total RNA was reverse transcribed into cDNA according to the instructions of a SYBR Premix Ex Taq ${ }^{\mathrm{TM}}$ II kit (Takara Bio, Inc.). The reaction conditions were as follows: $70^{\circ} \mathrm{C}$ for $5 \mathrm{~min}, 42^{\circ} \mathrm{C}$ for $60 \mathrm{~min}$, and $75^{\circ} \mathrm{C}$ for $10 \mathrm{~min}$. qPCR was carried out on a Roche LightCycler 480 instrument (Roche Diagnostics) using SYBRGreen reagent (Thermo Fisher Scientific, Inc.). The reaction conditions were as follows: Pre-denaturation at $95^{\circ} \mathrm{C}$ for $5 \mathrm{~min}$, and 40 cycles of denaturation at $95^{\circ} \mathrm{C}$ for $15 \mathrm{sec}$, annealing at $58^{\circ} \mathrm{C}$ for $35 \mathrm{sec}$, and extension at $72^{\circ} \mathrm{C}$ for $30 \mathrm{sec} . \beta$-actin was used as an internal reference, and relative gene expression was calculated using the $2^{-\Delta \Delta C q}$ method (23). Primer sequences are listed in Table I.

Western blot analysis. Total protein was extracted from mouse liver tissue using precooled RIPA buffer (Biosesang) containing protease inhibitor (Sigma-Aldrich; Merck KGaA), and the concentration was determined using a BCA kit (Beyotime Institute of Biotechnology). Then, $10 \mu \mathrm{g}$ protein was separated via SDS-PAGE on a $12 \%$ gel. The proteins were then transferred to a PVDF membrane, which was blocked with $5 \%$ non-fat milk for $1 \mathrm{~h}$ at room temperature. The membranes were incubated with primary antibodies against mammalian STE20-like protein kinase 1 (MST1; cat. no. ab79199; 1:500; $56 \mathrm{kDa}$ ), large tumour suppressor kinase 1 (LATS1; cat. no. ab70561; 1:5,000; $127 \mathrm{kDa}$ ), YAP (cat. no. ab52771; 1:5,000; $70 \mathrm{kDa}$ ) and TAZ (cat. no. ab119373; 1:1,000; $44 \mathrm{kDa}$ ) overnight at $4{ }^{\circ} \mathrm{C}$. A HRP-conjugated rabbit anti-mouse immunoglobulin $\mathrm{G}$ (cat. no. ab6728; 1:2,000) secondary antibody was added for $1 \mathrm{~h}$ at room temperature followed by exposure, development and visualization using an ECL working solution (EMD Millipore). $\beta$-actin (cat. no. ab8226; 1:1,000; $42 \mathrm{kDa}$ ) was used as an internal reference. All antibodies were purchased from Abcam. Protein bands were analysed using ImageJ2x software (version 2.1.4.7; National Institutes of Health).

Statistical analysis. SPSS 21.0 (IBM Corp.) was used for statistical analysis. The data were normally distributed, according to a Kolmogorov-Smirnov test. Thus, the results are presented as the mean \pm SD. Unpaired Student's t-tests were used to compare the differences between two groups. One-way 
A

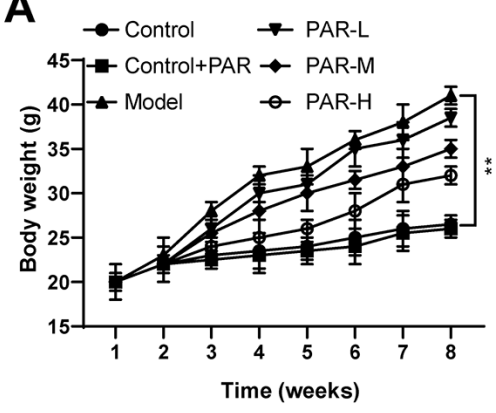

B

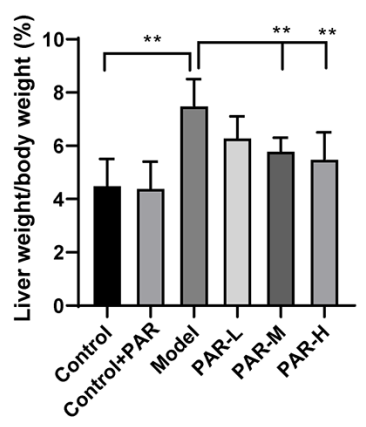

D

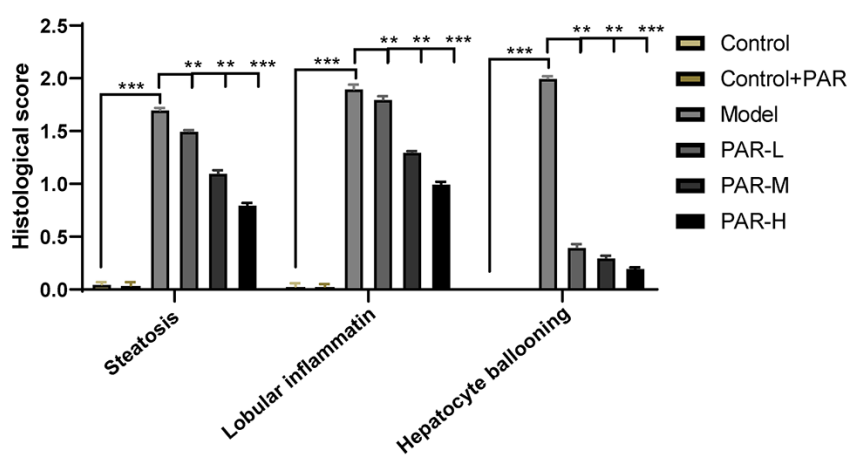

C

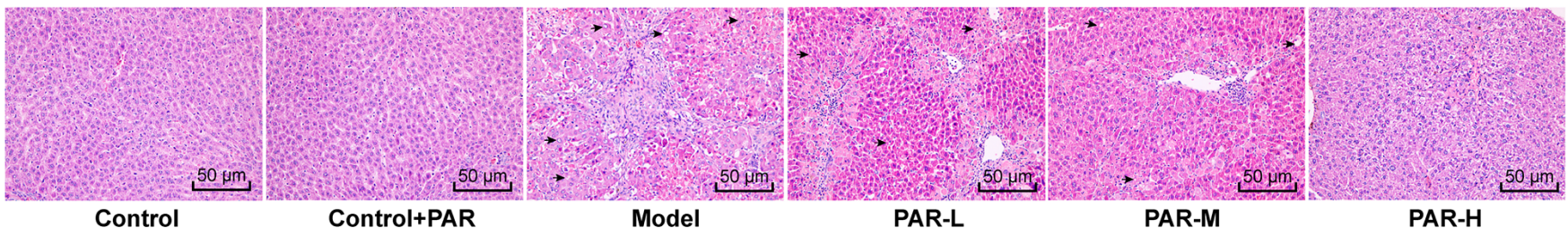

E

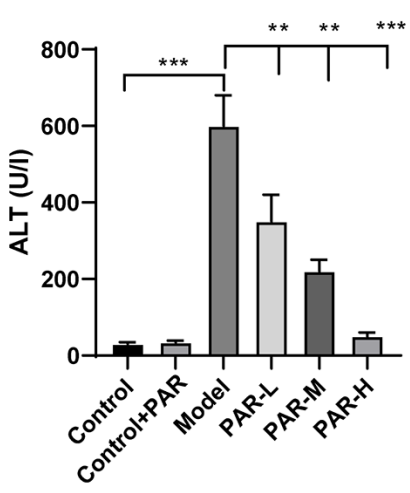

$\mathbf{F}$

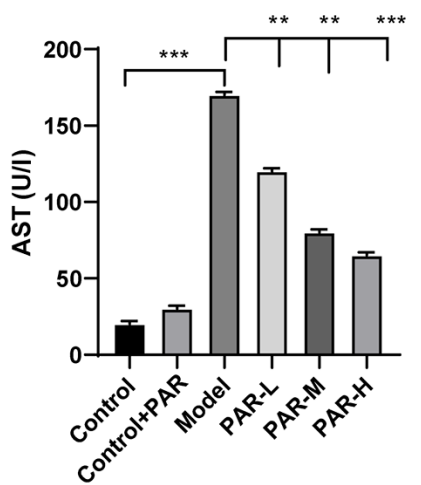

G

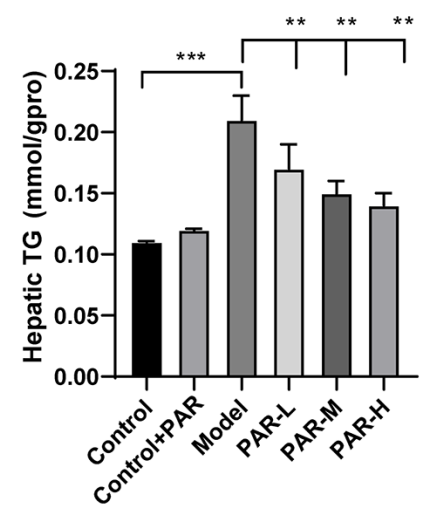

H

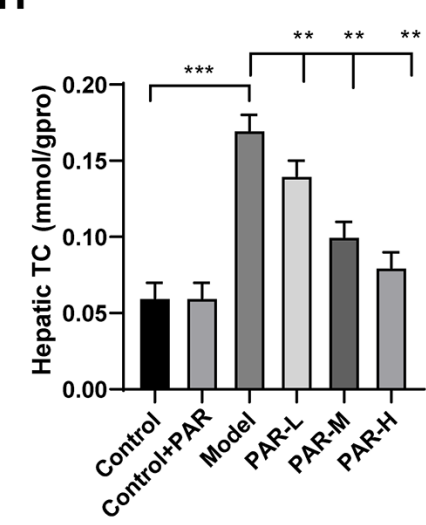

Figure 1. PAR has beneficial effects on the manifestations of MAFLD in mouse liver. (A) Changes in the body weight of mice during the experiment (1-8 weeks). ${ }^{* *} \mathrm{P}<0.01$, two-way ANOVA followed by Sidak's post hoc test. (B) Liver to body weight ratio at week 8 . (C) Representative images of H\&E staining of mouse livers (scale bar, $50 \mu \mathrm{m}$ ). (D) H\&E-stained sections were evaluated using a histological score. (E and F) ALT and AST serum levels in mice. ( $\mathrm{G}$ and $\mathrm{H}$ ) Hepatic TC and TG levels in mice. The data are presented as the mean \pm SD. $\mathrm{n}=6 .{ }^{* *} \mathrm{P}<0.01,{ }^{* * * *} \mathrm{P}<0.001$, one-way ANOVA followed by Sidak's post hoc test, unless otherwise stated. PAR, parthenolide; MFALD, metabolic dysfunction-associated fatty liver disease; H\&E, haematoxylin and eosin; ALT, alanine transaminase; AST, aspartate transaminase; TC, total cholesterol; TG, triglyceride; L, low; M, medium; H, high; gpro, gramme of protein.

or two-way ANOVA followed by Sidak's post hoc test were used to compare multiple groups. P-values were obtained from two-tailed tests. $\mathrm{P}<0.05$ was considered to indicate statistically significant difference.

\section{Results}

PAR alleviates liver injury in MAFLD mice. Previous studies have suggested that PAR has anti-inflammatory, antioxidant and antifibrotic properties (24-26). Therefore, it was hypothesized that PAR may have protective effects on MAFLD-induced liver injury. To examine effect of PAR on MAFLD, male C57BL/6 mice were fed high-fat diet for 8 weeks. The body weight and liver index (liver to body weight ratio) of mice in the model group were significantly higher than those in the control group $(\mathrm{P}<0.01$; Fig. 1A and $\mathrm{B})$. In addition, $H \& E$ staining demonstrated that hepatocytes in the model group displayed microbubbles and macrovesicular steatosis (Fig. 1C). The histological scores (steatosis, lobular inflammation and hepatocyte ballooning) in the model group were also significantly increased, compared with the control group (all $\mathrm{P}<0.01$; Fig. 1D). These results indicated that the MAFLD mouse model was successfully generated.

There were no significant differences in the body weight, liver index and H\&E staining in the liver sections between the control + PAR and the control groups (Fig. 1A-D), indicating that PAR did not damage normal mouse livers. Mice in the PAR-L, -M and -H treatment groups exhibited significantly reduced body weight, liver index and the histological score (all $\mathrm{P}<0.01$; Fig. 1A-D). PAR treatment also significantly reduced the serum levels of ALT and AST and decreased the hepatic $\mathrm{TC}$ and TG contents in MAFLD mice (all $\mathrm{P}<0.01$; Fig. 1E-H).

PAR improves liver lipid metabolism and fibrosis in MAFLD mice. Oil Red $\mathrm{O}$ staining was performed to determine whether PAR could improve hepatic lipid metabolism. The volume and number of lipid droplets were reduced in the PAR-H group, compared with the model group (Fig. 2A). 
A
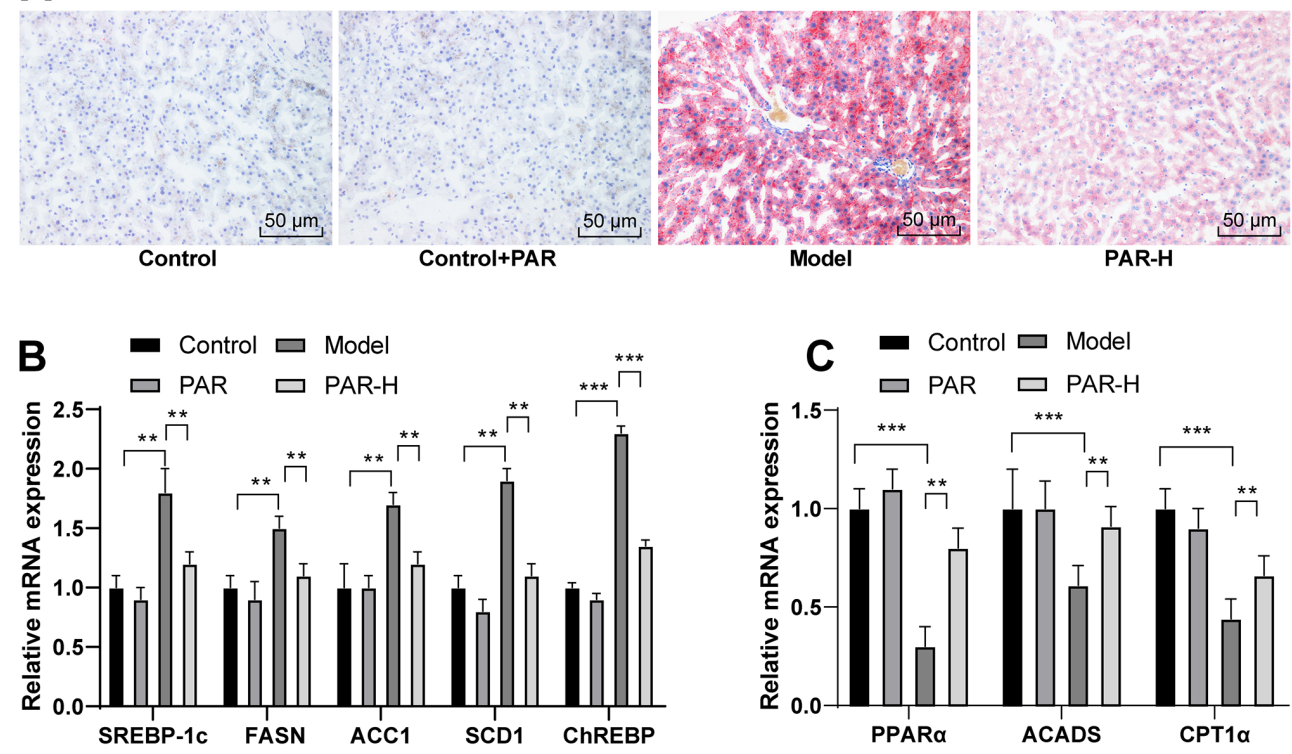

D
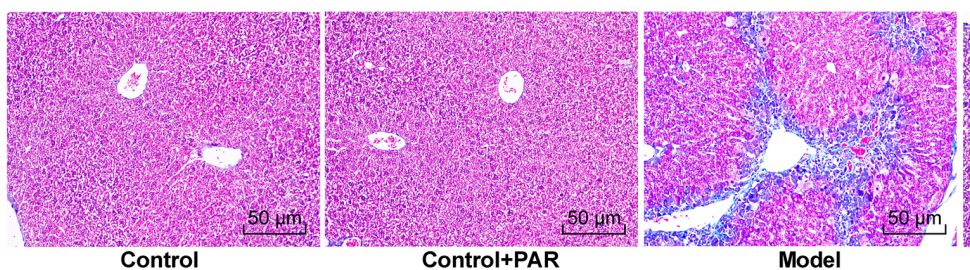

Model

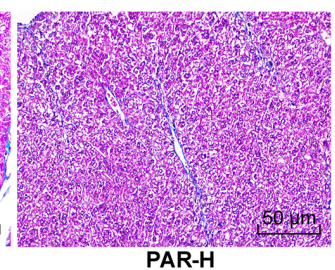

E
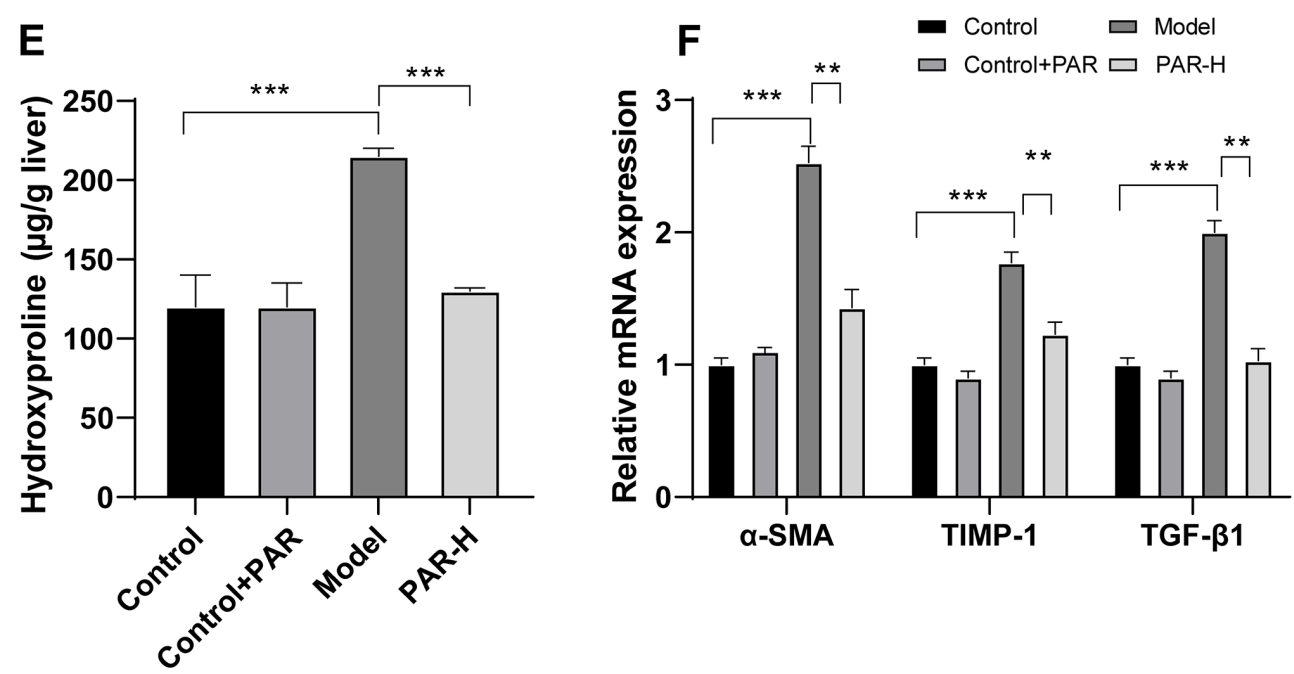

Figure 2. PAR improves hepatic lipid metabolism and fibrosis in mice with MAFLD. (A) Representative images of Oil Red O staining of mouse liver sections (scale bar, $50 \mu \mathrm{m}$ ). (B) mRNA expression levels of the liposynthesis-related genes SREBP-1c, FASN, ACC1, SCD1 and ChREBP. (C) mRNA expression levels of the fatty acid $\beta$-oxidation-related genes PPAR $\alpha$, ACADS and CPT1 $\alpha$. (D) Representative images of Masson's trichrome staining of mouse liver sections (scale bar, $50 \mu \mathrm{m}$ ). (E) Liver hydroxyproline content. ${ }^{* * *} \mathrm{P}<0.001$, one-way ANOVA followed by Sidak's post hoc test. (F) mRNA expression levels of hepatic fibrosis-related genes $\alpha$-SMA, TIMP- 1 and TGF- $\beta 1$. The data are presented as the mean \pm SD. $n=6 .{ }^{* * *} \mathrm{P}<0.01,{ }^{* * * *} \mathrm{P}<0.001$, two-way ANOVA followed by Sidak's post hoc test, unless otherwise stated. PAR, parthenolide; MFALD, metabolic dysfunction-associated fatty liver disease; SREBP-1c, sterol regulatory element binding protein-1c; FASN, fatty acid synthase; ACC1, acetyl CoA carboxylase 1; SCD1, stearoyl CoA desaturase 1; ChREBP, carbohydrate response element-binding protein; PPAR- $\alpha$; peroxisome proliferator-activated receptor $\alpha$; ACADS, acyl-CoA dehydrogenase short chain; CPT1 $\alpha$, carnitine palmitoyl transferase $1 \alpha$; $\alpha$-SMA, $\alpha$-smooth muscle actin; TIMP-1, tissue inhibitor of metalloproteinase 1; L, low; M, medium; $\mathrm{H}$, high.

Additionally, the expression levels of lipogenesis- and metabolism-related genes were measured using RT-qPCR. The mRNA expression levels of liposynthesis-related genes, such as sterol regulatory element binding protein (SREBP)-1c, fatty acid synthase (FASN), acetyl CoA carboxylase 1
(ACC1), stearoyl CoA desaturase 1 (SCD1) and carbohydrate response element-binding protein (ChREBP), were significantly lower in the PAR-H group than in the model group (all $\mathrm{P}<0.01$; Fig. 2B). Moreover, PAR-H treatment also significantly increased the mRNA expression levels of genes 

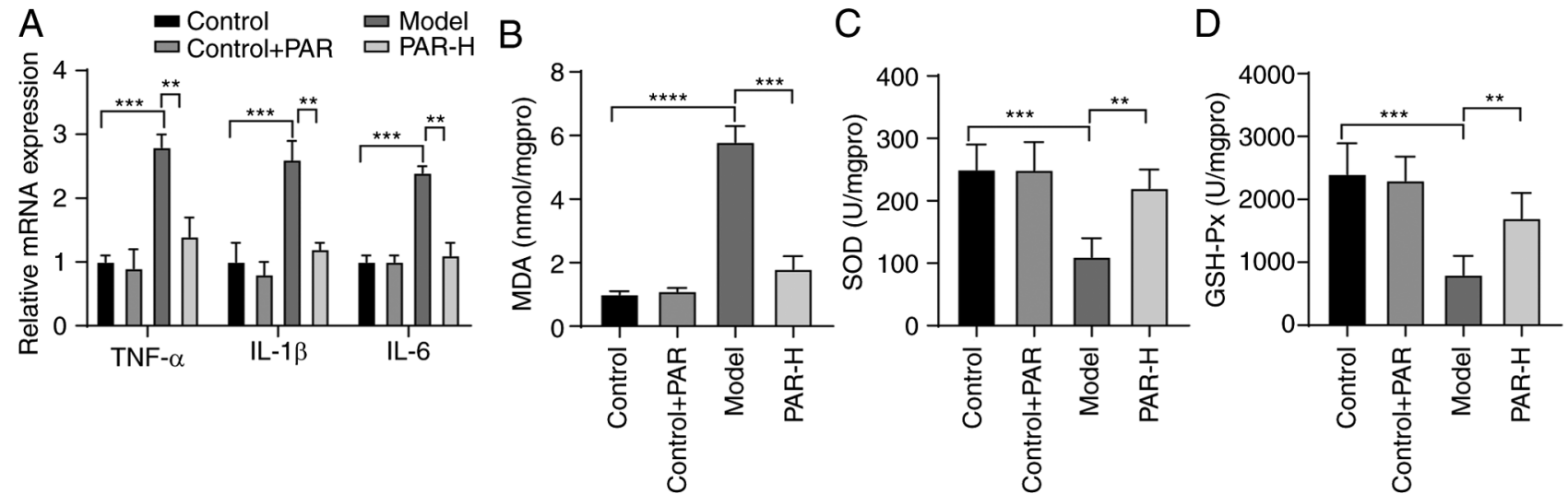

Figure 3. PAR reduces liver inflammation and oxidative stress in mice with MAFLD. (A) mRNA expression levels of pro-inflammatory cytokines TNF- $\alpha$, IL-1 $\beta$ and IL-6. ${ }^{* *} \mathrm{P}<0.01,{ }^{* * *} \mathrm{P}<0.001$, two-way ANOVA followed by Sidak's post hoc test. (B-D) MDA, SOD and GSH-Px levels in the liver. The data are presented as the mean \pm SD. $n=6 .{ }^{* *} \mathrm{P}<0.01,{ }^{* * *} \mathrm{P}<0.001$, two-way ANOVA followed by Sidak's post hoc test, unless otherwise stated. PAR, parthenolide; MFALD, metabolic dysfunction-associated fatty liver disease; MDA, malondialdehyde; SOD, superoxide dismutase; GSH-Px, glutathione peroxidase; H, high.
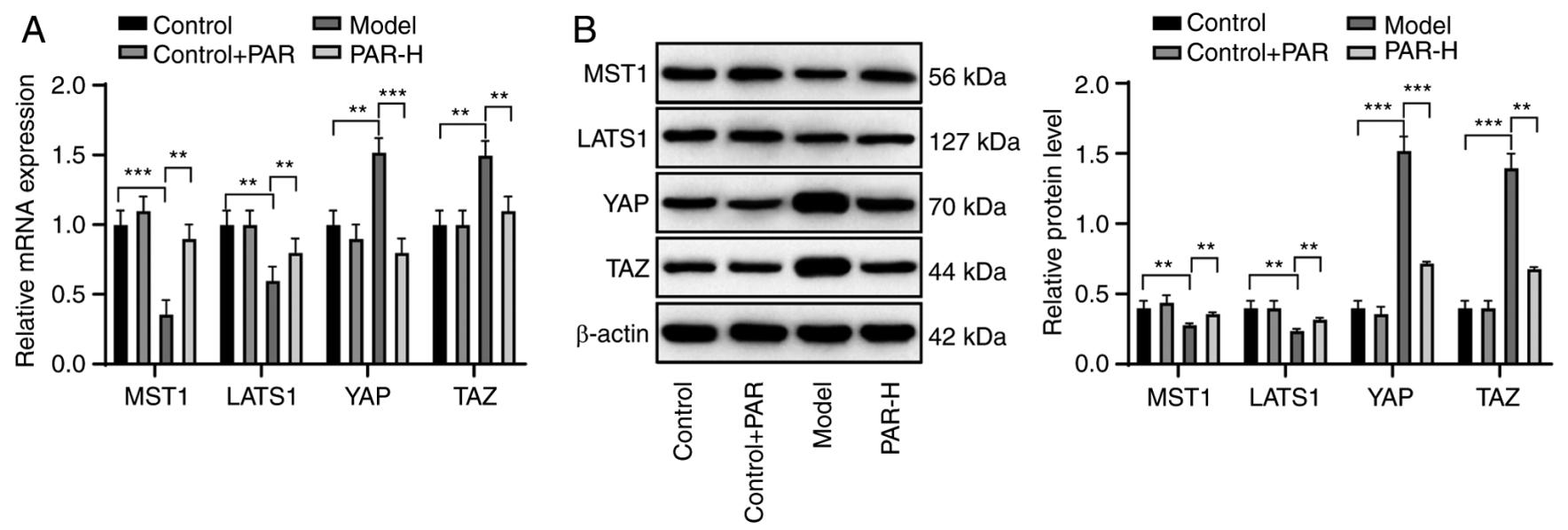

Figure 4. PAR activates the HIPPO pathway in the liver of mice with MAFLD. (A) mRNA and (B) protein expression levels of MST1, LATS1, YAP and TAZ were detected using reverse transcription-quantitative PCR and western blot analysis, respectively. The data are presented as the mean \pm SD. $n=6$. ${ }^{* *} \mathrm{P}<0.01$, ${ }^{* * *} \mathrm{P}<0.001$, two-way ANOVA followed by Sidak's post hoc test. PAR, parthenolide; MFALD, metabolic dysfunction-associated fatty liver disease; MST1, macrophage-stimulating 1; LATS1, large tumour suppressor kinase 1; YAP, Yes-associated protein; TAZ, transcriptional coactivator with PDZ-binding motif; low; M, medium.

associated with fatty acid $\beta$-oxidation, including peroxisome proliferator-activated receptor $\alpha$ (PPAR- $\alpha$ ), carnitine palmitoyl transferase $1 \alpha(\mathrm{CPT} 1 \alpha)$ and acyl-CoA dehydrogenase short chain (ACADS) (all P<0.01; Fig. 2C).

Liver fibrosis was assessed in all mice using Masson's trichrome staining, and liver hydroxyproline content was measured in order to determine whether PAR could reduce hepatic fibrosis in mice with MAFLD. Mice in the PAR-H group displayed improved liver fibrosis (Fig. 2D) and significantly reduced liver hydroxyproline content, compared with the model group $(\mathrm{P}<0.001$; Fig. $2 \mathrm{E})$. In addition, PAR-H treatment significantly reduced the mRNA expression levels of liver fibrosis-related genes, such as $\alpha$-smooth muscle actin $(\alpha-S M A)$, tissue inhibitor of metalloproteinase 1 (TIMP-1) and TGF- $\beta 1$ (all P<0.01; Fig. 2F).

$P A R$ reduces liver inflammation and oxidative stress in $M A F L D$ mice. The mRNA expression levels of pro-inflammatory cytokines (TNF- $\alpha$, IL-1 $\beta$ and IL-6) were measured using
RT-qPCR to determine the effect of PAR on liver inflammation in MAFLD. PAR-H treatment significantly reduced the expression levels of these pro-inflammatory factors in mice with MAFLD (all $\mathrm{P}<0.01$; Fig. 3A).

MDA levels and SOD and GSH-Px activities were measured in the liver in order to evaluate the role of PAR in hepatic oxidative stress in mice with MAFLD. Mice in the model group displayed a significant increase in MDA levels and a decrease in SOD and GSH-Px activities, compared with the control group (all $\mathrm{P}<0.001$; Fig. 3B-D). However, PAR-H treatment reversed these changes in mice with MAFLD (all $\mathrm{P}<0.01$; Fig. 3B-D).

PAR activates the HIPPO pathway in the liver of mice with $M A F L D$. YAP is a key mediator of the HIPPO pathway. Liver dysfunction is accompanied by an increase in the YAP expression in MAFLD and HIPPO pathway activation can reduce liver damage $(27,28)$. Therefore, it was hypothesized that PAR could play a protective role in the liver of mice with MAFLD 
A

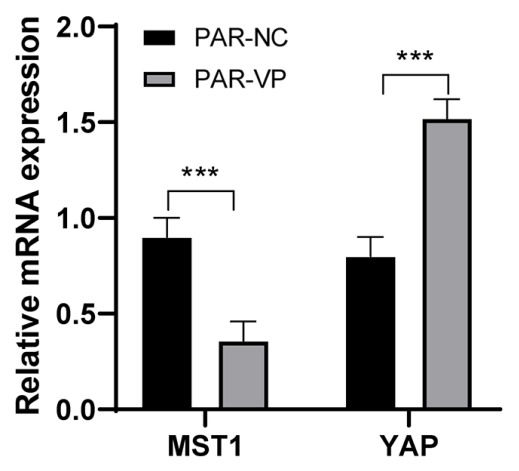

C

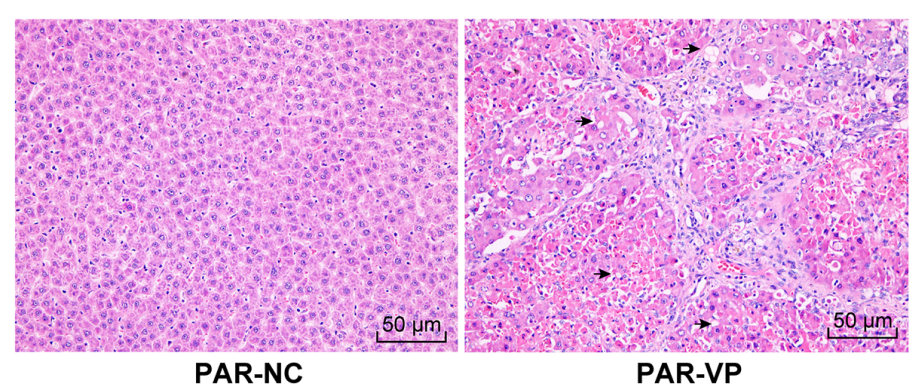

E

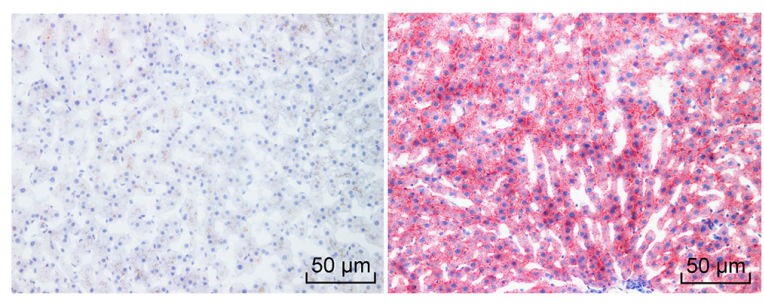

PAR-NC

F
B

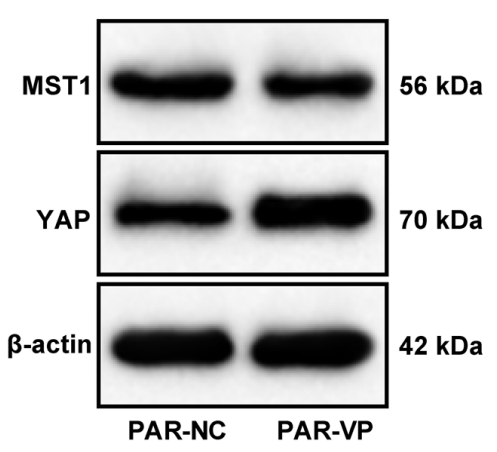

D
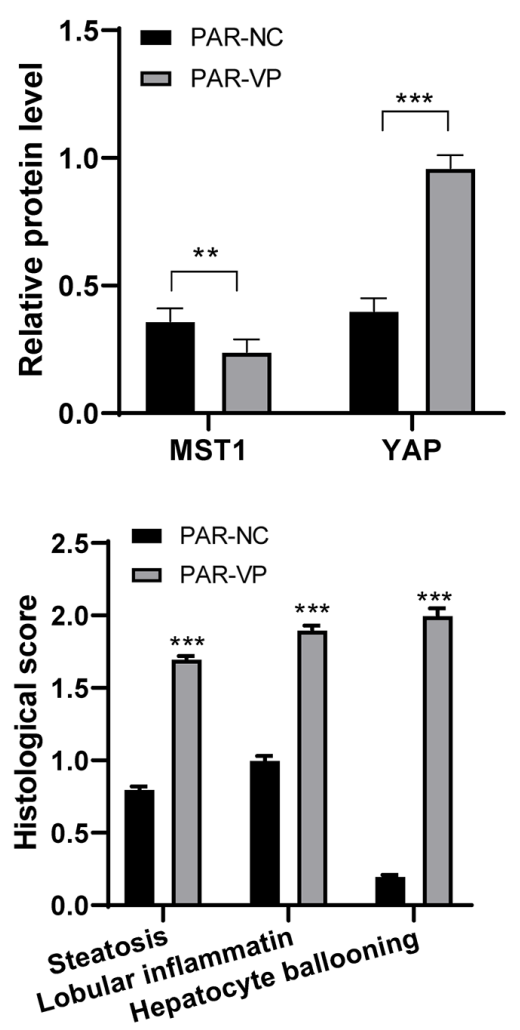

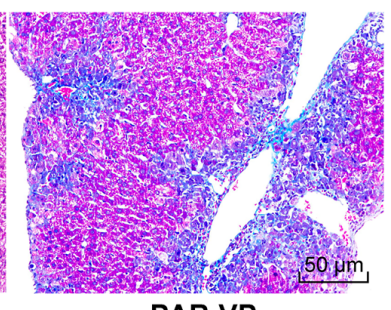

PAR-VP

Figure 5. Inhibition of the HIPPO pathway reverses the protective effect of PAR on the livers of mice with MAFLD. (A) mRNA and (B) protein expression levels of MST1 and YAP. (C) Representative images of H\&E staining of mouse livers (scale bar, $50 \mu \mathrm{m}$ ). (D) H\&E-stained sections were evaluated using a histological score. ${ }^{* * *} \mathrm{P}<0.001$, Student's t-test. (E) Representative images of Oil Red O staining of mouse liver sections (scale bar, $\left.50 \mu \mathrm{m}\right)$. (F) Representative images of Masson's trichrome staining of mouse liver sections. The data are presented as the mean \pm SD. $n=6 .{ }^{* * *} \mathrm{P}<0.01,{ }^{* * * * *} \mathrm{P}<0.001$ vs. PAR-NC group, two-way ANOVA followed by Sidak's post hoc test, unless otherwise stated. PAR, parthenolide; MFALD, metabolic dysfunction-associated fatty liver disease; H\&E, haematoxylin-eosin; VP, verteporfin; NC, negative control; MST1, macrophage-stimulating 1; YAP, Yes-associated protein.

through the activation of the HIPPO pathway. The mRNA and protein expression levels of several mediators of the HIPPO pathway (MST1, LATS1, YAP and TAZ) were measured. The expression levels of MST1 and LATS1 in the liver of PAR-H-treated mice were significantly higher than those in the model mice. Moreover, the expression levels of YAP and TAZ were reduced (all $\mathrm{P}<0.01$; Fig. $4 \mathrm{~A}$ and $\mathrm{B}$ ). These results indicated that PAR could activate the HIPPO pathway in the liver of mice with MAFLD.

Inhibition of the HIPPO pathway reverses the protective effect of PAR in the liver of mice with MAFLD. To confirm whether PAR treatment exerted beneficial effects on liver injury in mice with MAFLD through activation of the HIPPO pathway, mice were intraperitoneally injected with VP, an inhibitor of the HIPPO pathway. VP treatment in the PAR-VP group significantly reduced MST1 expression in the liver and increased YAP expression, compared with PAR-NC (all
$\mathrm{P}<0.01$; Fig. 5A and B), indicating that VP successfully inhibited the HIPPO pathway in vivo. H\&E staining demonstrated that hepatocytes in PAR-VP mice displayed microbubbles (Fig. 5C). In addition, the histological scores (steatosis, lobular inflammation and hepatocyte ballooning) in the PAR-VP group were significantly increased, compared with PAR-NC $(\mathrm{P}<0.001$; Fig. 5D). Moreover, PAR-VP treatment increased the number of lipid droplets (Fig. 5E) and aggravated hepatic fibrosis in mice (Fig. 5F).

\section{Discussion}

MAFLD is the leading cause of liver disease globally and affects both children and adults (29). PAR, a natural compound present in the feverfew plant, has various biological and therapeutic activities, including anti-inflammatory effects (30). In the present study, PAR attenuated the pathological symptoms of MAFLD in mice by activating the HIPPO pathway 
to suppress liver injury, hepatic lipid metabolism, fibrosis, inflammation and oxidative stress.

An increase in the serum levels of the biochemical markers AST, ALT, TC and TG may indicate acute hepatic failure (31). In the present study, PAR treatment improved histological scores and reduced the serum levels of ALT and AST, as well as TC and TG content in the liver of mice with MAFLD. Moreover, PAR administration reduced the body weight and the levels of TG, ALT and AST in the liver of MAFLD model mice, which was similar to the findings of a previous study (14). These results indicated that PAR exerted beneficial effects on liver injury in the MAFLD model.

A previous study has demonstrated that the lipid metabolism-associated genes SREBP-1c, ACC1, FASN, SCD1 and CPT1 $\alpha$ are markers of lipid accumulation and hepatic steatosis in MAFLD (32). ChREBP is an important transcription factor controlling hepatic energy metabolism and lipid metabolism (33). Reduced levels of hydroxyproline, as well as TGF- $\beta 1$, $\alpha$-SMA and TIMP-1 mRNA expression levels are closely associated with the suppression of hepatic fibrosis (34). The present findings demonstrated that PAR treatment downregulated the expression levels of liposynthesis markers (SREBP-1c, FASN, $\mathrm{ACC} 1, \mathrm{SCD} 1$ and ChREBP) and upregulated the expression of genes associated with fatty acid $\beta$-oxidation (PPAR $\alpha$, CPT $1 \alpha$ and ACADS). Similarly, PAR plays an inhibitory role in lipid accumulation and adipogenesis (30).

PAR normalizes the aberrant lipid metabolism and fatty acid $\beta$-oxidation in MAFLD rats (11). Additionally, a previous study has demonstrated that increased hydroxyproline content in the liver and hepatic collagen deposition are associated with aggravated liver fibrosis (35). The results of the present study indicated that PAR treatment could reduce the expression levels of liver fibrosis-associated markers $(\alpha-$ SMA, TIMP-1 and TGF- $\beta 1$ ), as well as liver hydroxyproline content and collagen deposition. These conclusions are supported by a study that demonstrated the protective effect of PAR on hepatic fibrosis (13). Overall, PAR alleviates the changes in hepatic lipid metabolism and fibrosis in mice with MAFLD.

Additionally, PAR has been reported to have potent anti-inflammatory and antioxidative stress activities $(14,36)$. TNF- $\alpha$, IL- $1 \beta$ and IL- 6 are proinflammatory cytokines (37). Decreased SOD and GSH-Px in combination with increased MDA levels are closely associated with oxidative stress (38). PAR has been shown to regulate obesity-induced inflammatory/oxidant responses and proposed as an effective agent for the treatment of obesity-related diseases (39). In agreement with these previous findings, PAR-treated MAFLD model mice in the present study displayed decreased TNF- $\alpha$, IL- $1 \beta$, IL-6 and MDA levels, together with elevated SOD and GSH-Px activities. Thus, PAR alleviated liver injury, changes in hepatic metabolism and fibrosis, inflammation and oxidative stress in the MAFLD model.

However, the mechanism of action of PAR in MAFLD is incompletely understood. The HIPPO pathway has been suggested to play a role in metabolic diseases through the regulation of key molecules, including MST1/2, LATS1/2 and the downstream YAP/TAZ transcriptional coactivators (40). The HIPPO pathway, as a novel pathway associated with growth control and cancer inhibition, plays a significantly regulatory role in liver development, injury and disease (41). The HIPPO pathway contributes to the prevention of MAFLD and liver cancer progression (42). Furthermore, a previous study has suggested that the HIPPO pathway is implicated in hepatic fibrosis (16). YAP is closely associated with the regulation of MAFLD-associated lipid metabolism and hepatic fibrosis (28). In the present study, PAR treatment activated the HIPPO pathway in MAFLD model mice, as evidenced by increased levels of MST1 and LATS1 expression and a reduction in the levels of YAP and TAZ expression. Additionally, inhibition of the HIPPO pathway reversed the protective effect of PAR in MAFLD model mice. However, previous studies have not reported that the protective effects of PAR in MAFLD are mediated by the HIPPO pathway, which was therefore a novel finding of the present study.

In conclusion, the present study demonstrated that PAR alleviated MAFLD through the activation of the HIPPO pathway in mice. However, only the protective effect of PAR on fatty liver in MAFLD was evaluated, and the role of PAR in other hepatic lesions was not studied. In the future, the role and mechanism of action of PAR in other types of liver injury will be further assessed. Altogether, the present study provided novel insight for the treatment of MAFLD; however, the clinical applications of these findings remain to be determined. Promising PAR-based therapeutic approaches involving the HIPPO pathway may also be investigated for the purpose of MAFLD treatment.

\section{Acknowledgements}

Not applicable.

\section{Funding}

No funding was received.

\section{Availability of data and materials}

The datasets used and/or analysed during the current study are available from the corresponding author on reasonable request.

\section{Authors' contributions}

WW, YH and QL conceived and designed the study. WW and QL performed the analysis and interpretation of the data and drafted the manuscript. YH and QL critically revised the article for important intellectual content and assisted in the literature search for this article. All authors agree to be accountable for all aspects of the work in ensuring that questions related to the accuracy or integrity of any part of the work are appropriately investigated. WW and YH confirm the authenticity of all the raw data. All authors read and approval the final manuscript.

\section{Ethics approval and consent to participate}

The present study was approved by The Animals Ethics Committee of Liaocheng People's Hospital (approval no. 2019036). Significant efforts were made to minimize the number of animals used and the pain they experienced. All procedures were conducted strictly in accordance with the 
National Institutes of Health Guide for the Care and Use of Laboratory Animals.

\section{Patient consent for publication}

Not applicable.

\section{Competing interests}

The authors declare that they have no competing interests.

\section{References}

1. Tilg $\mathrm{H}$ and Effenberger M: From NAFLD to MAFLD: When pathophysiology succeeds. Nat Rev Gastroenterol Hepatol 17: 387-388, 2020.

2. Farzanegi P, Dana A, Ebrahimpoor Z, Asadi M and Azarbayjani MA: Mechanisms of beneficial effects of exercise training on non-alcoholic fatty liver disease (NAFLD): Roles of oxidative stress and inflammation. Eur J Sport Sci 19: 994-1003, 2019.

3. Eslam M, Newsome PN, Sarin SK, Anstee QM, Targher G, Romero-Gomez M, Zelber-Sagi S, Wai-Sun Wong V, Dufour JF, Schattenberg JM, et al: A new definition for metabolic dysfunction-associated fatty liver disease: An international expert consensus statement. J Hepatol 73: 202-209, 2020.

4. Eslam M and George J: Reply to: Correspondence on 'A new definition for metabolic associated fatty liver disease: An international expert consensus statement': MAFLD: Moving from a concept to practice. J Hepatol 73: 1268-1269, 2020.

5. Eslam M, Valenti L and Romeo S: Genetics and epigenetics of NAFLD and NASH: Clinical impact. J Hepatol 68: 268-279, 2018.

6. Jegatheesan P and De Bandt JP: Fructose and NAFLD: The multifaceted aspects of fructose metabolism. Nutrients 9: 230, 2017.

7. Bellentani S: The epidemiology of non-alcoholic fatty liver disease. Liver Int 37 (Suppl 1): 81-84, 2017.

8. Rotman Y and Sanyal AJ: Current and upcoming pharmacotherapy for non-alcoholic fatty liver disease. Gut 66: 180-190, 2017.

9. Van De Wier B, Koek GH, Bast A and Haenen GR: The potential of flavonoids in the treatment of non-alcoholic fatty liver disease. Crit Rev Food Sci Nutr 57: 834-855, 2017.

10. Liu C, Liao JZ and Li PY: Traditional Chinese herbal extracts inducing autophagy as a novel approach in therapy of nonalcoholic fatty liver disease. World J Gastroenterol 23: 1964-1973, 2017.

11. Duan D, Zhang J, Yao J, Liu Y and Fang J: Targeting thioredoxin reductase by parthenolide contributes to inducing apoptosis of HeLa cells. J Biol Chem 291: 10021-10031, 2016.

12. Wang D, Wang H, Fu S, Cheng X, Yang F, Zhang Q, Li Y, Xue Z, Zhang L, Huang W, et al: Parthenolide ameliorates Concanavalin A-induced acute hepatitis in mice and modulates the macrophages to an anti-inflammatory state. Int Immunopharmacol 38: $132-138,2016$

13. Barbier-Torres L, Beraza N, Fernández-Tussy P, Lopitz-Otsoa F, Fernández-Ramos D, Zubiete-Franco I, Varela-Rey M, Delgado TC, Gutiérrez V, Anguita J, et al: Histone deacetylase 4 promotes cholestatic liver injury in the absence of prohibitin-1. Hepatology 62: 1237-1248, 2015.

14. Bahabadi M, Mohammadalipour A, Karimi J, Sheikh N, Solgi G, Goudarzi F, Hashemnia M and Khodadadi I: Hepatoprotective effect of parthenolide in rat model of nonalcoholic fatty liver disease. Immunopharmacol Immunotoxicol 39: 233-242, 2017.

15. Patel SH, Camargo FD and Yimlamai D: Hippo signaling in the liver regulates organ size, cell fate, and carcinogenesis. Gastroenterology 152: 533-545, 2017.

16. Zhou Y, Lv X, Qu H, Zhao K, Fu L, Zhu L, Ye G and Guo J: Differential expression of circular RNAs in hepatic tissue in a model of liver fibrosis and functional analysis of their target genes. Hepatol Res 49: 324-334, 2019.
17. Fujisawa K, Takami T, Sasai N, Matsumoto T, Yamamoto N and Sakaida I: Metabolic alterations in spheroid-cultured hepatic stellate cells. Int J Mol Sci 21: 3451, 2020.

18. Yi M, Li J, Chen S, Cai J, Ban Y, Peng Q, Zhou Y, Zeng Z, Peng S, Li X, et al: Correction to: Emerging role of lipid metabolism alterations in Cancer stem cells. J Exp Clin Cancer Res 37: 155,2018

19. Kodama T, Yi J, Newberg JY, Tien JC, Wu H, Finegold MJ, Kodama M, Wei Z, Tamura T, Takehara T, et al: Molecular profiling of nonalcoholic fatty liver disease-associated hepatocellular carcinoma using SB transposon mutagenesis. Proc Natl Acad Sci USA 115: E10417-E10426, 2018.

20. Lopez-Franco O,Hernández-Vargas P, Ortiz-Muñoz G,Sanjuán G, Suzuki Y, Ortega L, Blanco J, Egido J and Gómez-Guerrero C: Parthenolide modulates the NF-kappaB-mediated inflammatory responses in experimental atherosclerosis. Arterioscler Thromb Vasc Biol 26: 1864-1870, 2006.

21. Kim SL, Liu YC, Seo SY, Kim SH, Kim IH, Lee SO, Lee ST, Kim DG and Kim SW: Parthenolide induces apoptosis in colitis-associated colon cancer, inhibiting NF- $\kappa \mathrm{B}$ signaling. Oncol Lett 9: 2135-2142, 2015.

22. Kleiner DE, Brunt EM, Van Natta M, Behling C, Contos MJ, Cummings OW, Ferrell LD, Liu YC, Torbenson MS, Unalp-Arida A, et al: Design and validation of a histological scoring system for nonalcoholic fatty liver disease. Hepatology 41 : 1313-1321, 2005.

23. Livak KJ and Schmittgen TD: Analysis of relative gene expression data using real-time quantitative PCR and the 2(-Delta Delta C(T)) method. Methods 25: 402-408, 2001.

24. Wang M and Li Q: Parthenolide could become a promising and stable drug with anti-inflammatory effects. Nat Prod Res 29: 1092-1101, 2015.

25. Liao K, Xia B, Zhuang QY, Hou MJ, Zhang YJ, Luo B, Qiu Y, Gao YF, Li XJ, Chen HF, et al: Parthenolide inhibits cancer stem-like side population of nasopharyngeal carcinoma cells via suppression of the NF- $\mathrm{kB} / \mathrm{COX}-2$ pathway. Theranostics 5: 302-321, 2015.

26. Li XH, Xiao T, Yang JH, Qin Y, Gao JJ, Liu HJ and Zhou HG: Parthenolide attenuated bleomycin-induced pulmonary fibrosis via the NF- $\kappa$ B/Snail signaling pathway. Respir Res 19: 111, 2018.

27. Machado MV, Michelotti GA, Pereira TA, Xie G, Premont R, Cortez-Pinto H and Diehl AM: Accumulation of duct cells with activated YAP parallels fibrosis progression in non-alcoholic fatty liver disease. J Hepatol 63: 962-970, 2015.

28. Chen P, Luo Q, Huang C, Gao Q, Li L, Chen J, Chen B, Liu W, Zeng W and Chen Z: Pathogenesis of non-alcoholic fatty liver disease mediated by YAP. Hepatol Int 12: 26-36, 2018.

29. Younossi Z, Anstee QM, Marietti M, Hardy T, Henry L, Eslam M, George J and Bugianesi E: Global burden of NAFLD and NASH: Trends, predictions, risk factors and prevention. Nat Rev Gastroenterol Hepatol 15: 11-20, 2018.

30. Berdan CA, Ho R, Lehtola HS, To M, Hu X, Huffman TR, Petri Y, Altobelli CR, Demeulenaere SG, Olzmann JA, et al: Parthenolide covalently targets and inhibits focal adhesion kinase in breast cancer cells. Cell Chem Biol 26: 1027-1035 e22, 2019.

31. Wu CT, Deng JS, Huang WC, Shieh PC, Chung MI and Huang GJ: Salvianolic acid $C$ against acetaminophen-induced acute liver injury by attenuating inflammation, oxidative stress, and apoptosis through inhibition of the Keap1/Nrf2/HO-1 signaling. Oxid Med Cell Longev 2019: 9056845, 2019.

32. Xia SF, Shao J, Zhao SY, Qiu YY, Teng LP, Huang W, Wang SS, Cheng XR and Jiang YY: Niga-ichigoside F1 ameliorates high-fat diet-induced hepatic steatosis in male mice by Nrf2 activation. Food Funct 9: 906-916, 2018.

33. Rui L: Energy metabolism in the liver. Compr Physiol 4: 177-197, 2014.

34. Makled MN, Sharawy MH and El-Awady MS: The dual PPAR- $\alpha / \gamma$ agonist saroglitazar ameliorates thioacetamide-induced liver fibrosis in rats through regulating leptin. Naunyn Schmiedebergs Arch Pharmacol 392: 1569-1576, 2019.

35. Zhang Y, Miao H, Guan H, Wang C, Wang Z and Ji L: Long-term diosbulbin B treatment induced liver fibrosis in mice. Chem Biol Interact 298: 15-23, 2019. 
36. Li S, Gao X, Wu X, Wu Z, Cheng L, Zhu L, Shen D and Tong X: Parthenolide inhibits LPS-induced inflammatory cytokines through the toll-like receptor 4 signal pathway in THP-1 cells. Acta Biochim Biophys Sin (Shanghai) 47: 368-375, 2015.

37. Paolucci EM, Loukov D, Bowdish DME and Heisz JJ: Exercise reduces depression and inflammation but intensity matters. Biol Psychol 133: 79-84, 2018

38. Min YN, Niu ZY, Sun TT, Wang ZP, Jiao PX, Zi BB, Chen PP, Tian DL and Liu FZ: Vitamin E and vitamin $C$ supplementation improves antioxidant status and immune function in oxidative-stressed breeder roosters by up-regulating expression of GSH-Px gene. Poult Sci 97: 1238-1244, 2018

39. Kim CY, Jung YW and Park JS: Parthenolide, a feverfew-derived phytochemical, ameliorates obesity and obesity-induced inflammatory responses via the Nrf2/Keap1 pathway. Pharmacol Res 145: 104259, 2019.
40. Ardestani A, Lupse B and Maedler K: Hippo signaling: Key emerging pathway in cellular and whole-body metabolism. Trends Endocrinol Metab 29: 492-509, 2018.

41. Johnson RL: Hippo signaling and epithelial cell plasticity in mammalian liver development, homeostasis, injury and disease. Sci China Life Sci 62: 1609-1616, 2019.

42. Jeong SH, Kim HB, Kim MC, Lee JM, Lee JH, Kim JH, Kim JW, Park WY, Kim SY, Kim JB, et al: Hippo-mediated suppression of IRS2/AKT signaling prevents hepatic steatosis and liver cancer. J Clin Invest 128: 1010-1025, 2018.

This work is licensed under a Creative Commons Attribution-NonCommercial-NoDerivatives 4.0 International (CC BY-NC-ND 4.0) License. 